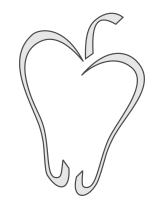

ISSN 1852-4249

\title{
Theory of terahertz Smith-Purcell radiation from a cylindrical grating
}

\author{
Z. Rezaei, ${ }^{1 *}$ B. Farokhi ${ }^{1}$
}

\begin{abstract}
An analysis of an annular electron beam propagating along a cylindrical grating with external magnetic field $B_{0}$ is presented. The grating comprises a dielectric in its slots. The dispersion relation of the modes is derived. The results demonstrate that the dielectric shifts the frequencies of the system modes to smaller values. The growth rates of the modes which are in phase with the beam are also considered. It is found that the decline in the growth rate is brought about by the dielectric. In addition, increasing the thickness of the dielectric and decreasing the height of the slots cause it to rise. The effect of beam thickness on growth rate is considered too. This is shown to increase and then fall as beam thickness increases. These results show that utilizing cylindrical grating loaded with dielectric has a promising effect on developing new kinds of compact high-efficient $\mathrm{THz}$ free-electron lasers based on Smith-Purcell radiation.
\end{abstract}

\section{Introduction}

Plasma and beam devices are employed in amplifiers, oscillators, charged particle accelerators, and high power sources of electromagnetic radiation. They are also used to transport electromagnetic energy and charged particles, and for basic plasma physics research [1].

When the electron beam passes near the grating surface (periodic structure), spontaneous radiations may be excited. This periodic structure can be a metallic corrugated surface with spatial periodicity $\mathrm{d}$ and corrugation height $\mathrm{h}$. This radiation was first observed by Smith and Purcell [2] in 1953. The Smith-Purcell radiation (SPR) is a tunable electromagnetic source, which is described by

\footnotetext{
*E-mail: z-rezaei@phd.araku.ac.ir
}

1 Department of Physics, Faculty of Science, Arak University, Arak, P.O. Box 38156-8-8349, Iran

$$
\lambda=\frac{d}{n}\left(\frac{1}{\beta}-\cos \theta\right),
$$

where $\lambda$ is the wavelength of the radiated wave from the grating, $n$ is the order of this radiation, $\beta$ is the relative velocity of the charge, and $\theta$ is the direction of the radiated wave with respect to this charge.

In the far-infrared or terahertz $(\mathrm{THz})$ region, several theories have been proposed to describe the operation of SPR, and also its application, in a free electron laser (FEL). Schachter and Ron proposed a theory based on the interaction of an electron beam with a wave traveling along the grating. They used some approximation to evaluate the reflection matrix of the grating and found a cubic equation for growth rate, which is consistent with Cherenkov FEL [3]. Urata et al. considered this phenomenon experimentally, and observed high power coherent superradiant SP emission in the far infrared $(30-100 \mu \mathrm{m})$ region [4]. SP radiation in the ultraviolet and near infrared regions was also detected by Y. Neo et al. [5]. Kim and Song, using the interaction of the electrons with a traveling wave, solved the initial problem of the sheet-beam and found a 
Papers in Physics, vol. 11, ART. 110007 (2019) / Z. Rezaei et al.

quadratic equation for the exponential growth rate [6]. Later Andrews and Brau explained Urata's experiment as bunching of the beam electrons due to the interaction of an evanescent wave with this sufficiently high-current beam. They also derived the gain of this radiation, which had cube root dependence on the beam current [7]. Also in 2004, Freund et al. [8] developed a linear theory of a gratingcoupled Smith-Purcell traveling wave in a parallel plate wave guide. They found the linearized dispersion relation for the vacuum structures and the wave-particle interaction in an arbitrary magnetic field. Then, D. Li et al. performed simulation and confirmed the theory of Andrews and coworkers about the mechanism of superradiation, which happens at integer multiples of the bunching frequency [9]. In addition, the growth rate of SP-FEL was considered by D. N. Klochkov et al. and found to be proportional to the square root of the sheet electron beam current [10].

Loading dielectric is an important physical mechanism which has been successfully applied to some high power microwave and terahertz systems. A dielectric-loaded grating for 3D Smith-Purcell rectangular device is proposed by Cao et al. [11,12]. They used 3D particle-in-cell simulation to find the dispersion relations at the operating point, and the growth rates. W. Liu et al. considered a rectangular grating filled with dielectric. They obtained the minimum current for starting the SP oscillator and deduced that the dielectric will decrease this current. Also, they explained the effect of changing the beam parameters on the growth rate [13].

There is no edge effect issue in cylindrical grating driven by an annular beam. Therefore, as the cylindrical gratings are more efficient, with fewer losses than rectangular ones, they are more applicable in radiation sources and considered in many types of research. H. P. Bluem et al. worked on a cylindrical grating exposed by an annular beam. They observed both superradiance and SP radiation [14]. S. Hasegawa et al. considered a cylindrical corrugation in a waveguide. They reported BWO operation, excited by a cylindrical surface wave in k-band signal region. Also, they increased the voltage of the beam and observed SP radiation in the u-band and E-band of frequency, which was the result of interaction between the higher modes of the waveguide [15].

Here we present a linear theory of an annular

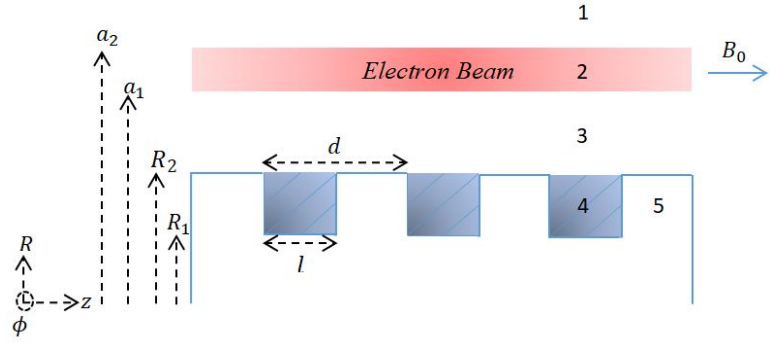

Figure 1: The cross section view of the grating, filled with dielectric. In addition, the annular electron beam is drifting along the axial direction with an externally magnetic field $B_{0}$.

electron beam, magnetized, propagating along a cylindrical metallic grating. The slots of this grating are filled with dielectric. The results of this paper highlight the basic problem of developing SPFEL based on cylindrical grating loaded with dielectric. For simplicity, we assume that the system is uniform in the direction parallel to the slots of the grating. The fundamental dynamical equations are presented in section II. The results and discussion are given in section III. The conclusions are considered at the end.

\section{Theory}

Consider a cylindrical grating which is made of an ideal metal. The inner and outer radii of the grating are $R_{1}$ and $R_{2}$, respectively as illustrated in Fig. 1. As shown in this figure, $d$ denotes the period of the grating, $l$ is the length of the slot openings (which will be filled with dielectric) and $h$ is the depth of the slots $\left(R_{2}-R_{1}\right)$. An annular electron beam with inner radius $a_{1}$ and outer radius $a_{2}$ $=a_{1}+\Delta$ in a uniform static axial magnetic field $B_{0}$ is drifting with velocity $v_{0}$ along the axis of the grating and very close to it. We assume that there is no transverse disturbed movement in the electron beam. In addition, for simplicity, we assume that the system is uniform in the $\varphi$ direction.

\section{i. Dispersion relation}

The dispersion relation of the modes of this system is the result of considering Maxwell's equations with the continuity equation and the relativistic 
momentum equation for electron beams:

$$
\begin{aligned}
& \boldsymbol{\nabla} \times \boldsymbol{E}=-\frac{\partial \boldsymbol{B}}{\partial t}, \\
& \boldsymbol{\nabla} \times \boldsymbol{B}=\mu \boldsymbol{J}+\frac{1}{c^{2}} \frac{\partial \boldsymbol{E}}{\partial t} \\
& \frac{\partial n}{\partial t}+\nabla \cdot(n \boldsymbol{v})=0 \\
& \gamma^{3} m_{0} n_{0}\left[\frac{\partial}{\partial t} \boldsymbol{v}+\boldsymbol{v}_{0} \cdot \boldsymbol{\nabla} \boldsymbol{v}\right]=-e n_{0}\left(\boldsymbol{E}_{z}+\boldsymbol{v} \times \boldsymbol{B}\right) .
\end{aligned}
$$

As a consequence, we expand all quantities in terms of an unperturbed part plus a small perturbation as follows: $n=n_{0}+\delta n, \boldsymbol{v}=\boldsymbol{v}_{0}+\boldsymbol{\delta} \boldsymbol{v}_{z}, \boldsymbol{J}=\boldsymbol{J}_{0}+\boldsymbol{\delta} \boldsymbol{J}$, $\boldsymbol{E}=\boldsymbol{\delta} \boldsymbol{E}$ and $\boldsymbol{B}=B_{0} \hat{\boldsymbol{z}}+\boldsymbol{\delta} \boldsymbol{B}$, where $n$ and $v$ are the electron density and velocity, respectively. Unperturbed beam density, $n_{0}$, is uniform and timeindependent, $\boldsymbol{\delta} \boldsymbol{E}$ and $\boldsymbol{\delta} \boldsymbol{B}$ are the electric and magnetic fields, $\boldsymbol{J}=$ en $\boldsymbol{v}$ is the density of current, $\gamma=\left(1-v^{2} / c^{2}\right)^{1 / 2}$ is the relativistic factor, and $c$ is the velocity of light in free space.

The perturbed density of current is

$$
\boldsymbol{\delta} \boldsymbol{J}=-e\left(n \boldsymbol{\delta} \boldsymbol{v}_{z}+\delta n \boldsymbol{v}_{0}\right)
$$

which, with the help of the continuity and momentum equations is defined as

$$
\boldsymbol{\delta} \boldsymbol{J}=\frac{i \omega \varepsilon_{0}}{\gamma^{3}} \frac{\omega_{p}^{2}}{\left(\omega-k_{n} v_{0}\right)^{2}} \boldsymbol{\delta} \boldsymbol{E}_{z} .
$$

Here, $\omega_{p}^{2}=\frac{n e^{2}}{m_{0} \varepsilon_{0}}$ is the relativistic beam plasma frequency.

The fields in the electron beam part will be derived from this wave equation

$$
\nabla^{2} \boldsymbol{\delta} \boldsymbol{B}+\frac{\omega^{2}}{c^{2}} \varepsilon^{\Pi} \boldsymbol{\delta} \boldsymbol{B}=0,
$$

where $\varepsilon^{\Pi}=1-\frac{\omega_{p}^{2}}{\gamma^{3}\left(\omega-k_{n} v_{0}\right)^{2}}$ is the relative dielectric constant in the electron beam [12].

Also, in region 1 and 3, where there is no electron beam, the electromagnetic fields are the solution of the wave equation of this form

$$
\left(\nabla_{\perp}^{2}+\beta_{n}^{2}\right)\left\{\begin{array}{l}
\delta E_{z} \\
\delta B_{z}
\end{array}=0 .\right.
$$

We suppose that the TM mode propagates in this device. And, by applying Floquet's theorem, the radiation fields take the general form

$$
\boldsymbol{\delta} \boldsymbol{f}(\boldsymbol{r}, t)=\sum_{n=0}^{\infty} \boldsymbol{\delta} \boldsymbol{f}_{n}(r) e^{i\left(k_{n} z-\omega t\right)},
$$

where $\boldsymbol{f}_{n}, k_{n}=k_{0}+\frac{2 n \pi}{d}$, and $\omega$ represent the Fourier coefficient, wave number in the axial direction and frequency of the nth mode, respectively.

\section{Region 1}

This region is the vacuum above the electron beam. So the fields in $a_{2}<r$ can be expressed as below

$$
\begin{aligned}
& E_{z}(r, z)=\sum_{n=-\infty}^{\infty} b_{n} K_{0}\left(\beta_{n} r\right) e^{i\left(k_{n} z-\omega t\right)}, \\
& B_{\varphi}(r, z)=\sum_{n=-\infty}^{\infty} \frac{i \omega}{c^{2} \beta_{n}} n_{n} K_{1}\left(\beta_{n} r\right) e^{i\left(k_{n} z-\omega t\right)} .
\end{aligned}
$$

\section{Region 3}

In this region, $R_{2}<r<a_{1}$, the fields are

$$
\begin{aligned}
& E_{z}(r, z)= \\
& \quad \sum_{n=-\infty}^{\infty}\left[c_{n} l_{0}\left(\beta_{n} r\right)+d_{n} K_{0}\left(\beta_{n} r\right)\right] e^{i\left(k_{n} z-\omega t\right)} \\
& B_{\varphi}(r, z)= \\
& \quad \sum_{n=-\infty}^{\infty} \frac{-i \omega}{c^{2} \beta_{n}}\left[c_{n} l_{1}\left(\beta_{n} r\right)-d_{n} K_{1}\left(\beta_{n} r\right)\right] e^{i\left(k_{n} z-\omega t\right)} .
\end{aligned}
$$

Region 2

In the electron beam region, $a_{1}<r<a_{2}$, the evanescent waves are the solution of Eq. (5), and have the following forms

$$
\begin{aligned}
& E_{z}(r, z)= \\
& \quad \sum_{n=-\infty}^{\infty} \frac{i}{r \omega \varepsilon_{0} \varepsilon^{\Pi}}\left\{g_{n}\left[r \kappa_{1 n} l_{1}\left(\kappa_{1 n} r\right)+l_{0}\left(\kappa_{1 n} r\right)\right]\right. \\
& \left.\quad+f_{n}\left[-r \kappa_{1 n} K_{1}\left(\kappa_{1 n} r\right)+K_{0}\left(\kappa_{1 n} r\right)\right]\right\} e^{i\left(k_{n} z-\omega t\right)}, \\
& B_{\varphi}(r, z)= \\
& \quad \sum_{n=-\infty}^{\infty} \mu\left\{g_{n} l_{0}\left(\kappa_{1 n} r\right)+f_{n} K_{0}\left(\kappa_{1 n} r\right)\right\} e^{i\left(k_{n} z-\omega t\right)} .
\end{aligned}
$$

Where, $\kappa_{1 n}=\sqrt{k_{n}^{2}-\omega^{2} \varepsilon^{\Pi} / c^{2}}$. 
Regions 4 and 5

The slot openings (region 4) are filled with dielectric $\varepsilon_{r}$. The solutions of the wave equation in this region are

$$
\begin{aligned}
E_{z}(r, z)= & \sum_{m=0}^{\infty} e_{m}\left[H_{0}\left(\tau_{m} r\right)\right. \\
& \left.-\frac{H_{0}\left(\tau_{m} R_{1}\right)}{G_{0}\left(\tau_{m} R_{1}\right.} G_{0}\left(\tau_{m} r\right)\right] \cos \left(\frac{m \pi}{l} z\right), \\
B_{\varphi}(r, z)= & \sum_{m=0}^{\infty} e_{m} \frac{i \omega \varepsilon_{r}}{c^{2} \tau_{m}}\left[\dot{H}_{0}\left(\tau_{m} r\right)\right. \\
& \left.-\frac{H_{0}\left(\tau_{m} R_{1}\right)}{G_{0}\left(\tau_{m} R_{1}\right.} \dot{G}_{0}\left(\tau_{m} r\right)\right] \cos \left(\frac{m \pi}{l} z\right) .
\end{aligned}
$$

Where,

$$
\begin{aligned}
& H_{0}\left(\tau_{m} r\right)=\left\{\begin{array}{l}
J_{0}\left(\tau_{m} r\right) \\
l_{0}\left(\dot{\tau}_{m} r\right)
\end{array},\right. \\
& G_{0}\left(\tau_{m} r\right)=\left\{\begin{array}{l}
N_{0}\left(\tau_{m} r\right) \\
K_{0}\left(\dot{\tau}_{m} r\right)
\end{array},\right. \\
& \dot{H}_{0}\left(\tau_{m} r\right)=\left\{\begin{array}{c}
-J_{1}\left(\tau_{m} r\right) \\
l_{1}\left(\dot{\tau}_{m} r\right)
\end{array},\right. \\
& \dot{G}_{0}\left(\tau_{m} r\right)=\left\{\begin{array}{l}
-N_{1}\left(\tau_{m} r\right) \\
-K_{1}\left(\dot{\tau}_{m} r\right)
\end{array},\right. \\
& k_{z}=\frac{m \pi}{l}, \quad \tau_{m}=\sqrt{\varepsilon_{r} \frac{\omega^{2}}{c^{2}}-k_{z}^{2}}>0, \\
& \dot{\tau}_{m}=\sqrt{k_{z}^{2}-\varepsilon_{r} \frac{\omega^{2}}{c^{2}}}>0 .
\end{aligned}
$$

As we assumed $k_{0} d<2 \pi$, it is enough to keep just one mode $(m=0)$ in the slots, so the standing waves will be the fields in this part of the system. Also, there is no field in the ideal metal of region 5.

After applying the continuity conditions for the fields in the border of regions $1-2,2-3$ and $3-4$, the dispersion relation will be as below

$$
R\left(\omega, k_{n}, \varepsilon^{\Pi}\right)=0 .
$$

In which,

$$
\begin{aligned}
& R\left(\omega, k_{n}, \varepsilon^{\Pi}\right)=\sum_{n=-\infty}^{\infty}\left\{\frac{-1}{k_{n}^{2} \beta_{n} d}\left[2-2 \cos \left(k_{n} l\right)\right]\right. \\
& \times\left[H_{0}\left(\tau_{0} R_{2}\right)-\frac{H_{0}\left(\tau_{0} R_{1}\right)}{G_{0}\left(\tau_{0} R_{1}\right)} G_{0}\left(\tau_{0} R_{2}\right)\right] \\
& \times \frac{\alpha_{a} I_{1}\left(\beta_{n} R_{2}\right)+\alpha_{b} K_{1}\left(\beta_{n} R_{2}\right)}{\alpha_{a} I_{0}\left(\beta_{n} R_{2}\right)-\alpha_{b} K_{0}\left(\beta_{n} R_{2}\right)} \\
& \left.-\frac{\varepsilon_{r} l}{\tau_{0}}\left[\dot{H}_{0}\left(\tau_{0} R_{2}\right)-\frac{H_{0}\left(\tau_{0} R_{1}\right)}{G_{0}\left(\tau_{0} R_{1}\right)} \dot{G}_{0}\left(\tau_{0} R_{2}\right)\right]\right\} \text {, } \\
& \alpha_{a}=-\alpha_{1} \alpha_{6}+\alpha_{2} \alpha_{5}, \quad \alpha_{b}=-\alpha_{3} \alpha_{6}+\alpha_{4} \alpha_{5} \text {, } \\
& \alpha_{1}=\frac{K_{1}\left(\beta_{n} a_{1}\right)}{\beta_{n} \varepsilon^{\Pi} a_{1}}\left[a_{1} \kappa_{1 n} I_{1}\left(\kappa_{1 n} a_{1}\right)+I_{0}\left(\kappa_{1 n} a_{1}\right)\right] \\
& +K_{0}\left(\beta_{n} a_{1}\right) I_{0}\left(\kappa_{1 n} a_{1}\right), \\
& \alpha_{2}=\frac{K_{1}\left(\beta_{n} a_{1}\right)}{\beta_{n} \varepsilon^{\Pi} a_{1}}\left[-a_{1} \kappa_{1 n} K_{1}\left(\kappa_{1 n} a_{1}\right)+K_{0}\left(\kappa_{1 n} a_{1}\right)\right] \\
& +K_{0}\left(\beta_{n} a_{1}\right) K_{0}\left(\kappa_{1 n} a_{1}\right) \text {, } \\
& \alpha_{3}=-\frac{I_{1}\left(\beta_{n} a_{1}\right)}{\beta_{n} \varepsilon^{\Pi} a_{1}}\left[a_{1} \kappa_{1 n} I_{1}\left(\kappa_{1 n} a_{1}\right)+I_{0}\left(\kappa_{1 n} a_{1}\right)\right] \\
& +I_{0}\left(\beta_{n} a_{1}\right) I_{0}\left(\kappa_{1 n} a_{1}\right), \\
& \alpha_{4}=-\frac{I_{1}\left(\beta_{n} a_{1}\right)}{\beta_{n} \varepsilon^{\Pi} a_{1}}\left[-a_{1} \kappa_{1 n} K_{1}\left(\kappa_{1 n} a_{1}\right)+K_{0}\left(\kappa_{1 n} a_{1}\right)\right] \\
& +I_{0}\left(\beta_{n} a_{1}\right) K_{0}\left(\kappa_{1 n} a_{1}\right) \text {, } \\
& \alpha_{5}=\frac{K_{1}\left(\beta_{n} a_{2}\right)}{\beta_{n} \varepsilon^{\Pi} a_{2}}\left[a_{2} \kappa_{1 n} I_{1}\left(\kappa_{1 n} a_{2}\right)+I_{0}\left(\kappa_{1 n} a_{2}\right)\right] \\
& +K_{0}\left(\beta_{n} a_{2}\right) I_{0}\left(\kappa_{1 n} a_{2}\right), \\
& \alpha_{6}=\frac{K_{1}\left(\beta_{n} a_{2}\right)}{\beta_{n} \varepsilon^{\Pi} a_{2}}\left[-a_{2} \kappa_{1 n} K_{1}\left(\kappa_{1 n} a_{2}\right)+K_{0}\left(\kappa_{1 n} a_{2}\right)\right] \\
& +K_{0}\left(\beta_{n} a_{2}\right) K_{0}\left(\kappa_{1 n} a_{2}\right) \text {. }
\end{aligned}
$$

If there is no beam $\left(\alpha_{1}=\alpha_{5}, \alpha_{2}=\alpha_{6}\right)$, the dispersion relation will become as

$$
\begin{gathered}
R\left(\omega_{0}, k_{0}, 1\right)=\sum_{n=-\infty}^{\infty}\left\{\frac{1}{k_{n}^{2} \beta_{n} d}\left[2-2 \cos \left(k_{n} l\right)\right]\right. \\
\times\left[H_{0}\left(\tau_{0} R_{2}\right)-\frac{H_{0}\left(\tau_{0} R_{1}\right)}{G_{0}\left(\tau_{0} R_{1}\right)} G_{0}\left(\tau_{0} R_{2}\right)\right] \frac{K_{1}\left(\beta_{n} R_{2}\right)}{K_{0}\left(\beta_{n} R_{2}\right)} \\
\left.-\frac{\varepsilon_{r} l}{\tau_{0}}\left[H_{0}\left(\tau_{0} R_{2}\right)-\frac{H_{0}\left(\tau_{0} R_{1}\right)}{G_{0}\left(\tau_{0} R_{1}\right)} G_{0}\left(\tau_{0} R_{2}\right)\right]\right\} \\
=0 .
\end{gathered}
$$

This is similar to the dispersion relation in [14] and [16] in the limit of $\varepsilon_{r}=1$. 

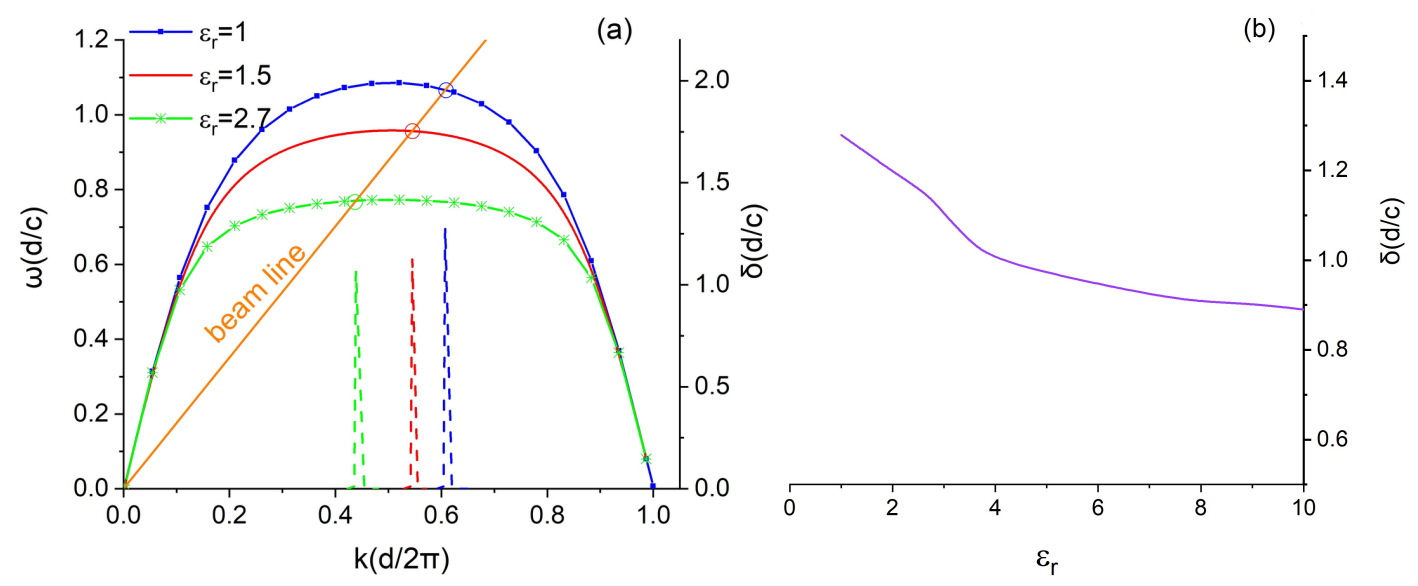

Figure 2: (a) Comparison of dispersion relations for different $\varepsilon_{r} s$ (solid curves). The beam line with voltage $20 \mathrm{kev}$ is also plotted for reference. The growth rate $(\delta)$ for each curve, near the intersection (the circles), is indicated by a dash line. (b) The effect of relative dielectric $\varepsilon_{r}$ on the growth rate.

\section{ii. Growth rate}

So far, the dispersion relation of the modes in this configuration has been derived. One of these modes can grow if it is in resonance with the electron beam. So, we assume that the frequency of this mode is $\omega=\omega_{r}+\delta$. Then the Taylor expansion of the dispersion relation about the synchronous point $\left(\omega_{r}, k_{r}\right)$ will become

$$
\begin{aligned}
R\left(\omega, k_{n}, \varepsilon^{\Pi}\right) & =R\left(\omega_{r}, k_{r}, 1\right) \\
& +\left.\left(\omega-\omega_{r}\right) \frac{\partial R\left(\omega, k_{n}, \varepsilon^{\Pi}\right)}{\partial \omega}\right|_{\left(\omega_{r}, k_{r}, 1\right)} \\
& +\left.\left(\varepsilon^{\Pi}-1\right) \frac{\partial R\left(\omega, k_{n}, \varepsilon^{\Pi}\right)}{\partial \varepsilon^{\Pi}}\right|_{\left(\omega_{r}, k_{r}, 1\right)} .
\end{aligned}
$$

By assuming that $\delta$ is small, the equation below will be found

$$
\begin{aligned}
\left(R_{0} x^{2}-\frac{\omega_{p}^{2}}{\gamma^{3}} \dot{R}_{\varepsilon \Pi}\right)+\left(2 x R_{0}\right. & \left.+x^{2} \dot{R}_{\omega}\right) \delta \\
& +2 x \dot{R}_{\omega} \delta^{2}=0 .
\end{aligned}
$$

In which,

$$
\begin{aligned}
x & =\omega_{r}-k_{r} v, \\
\dot{R}_{\varepsilon^{\Pi}} & =\left.\frac{\partial R\left(\omega, k_{n}, \varepsilon^{\Pi}\right)}{\partial \varepsilon^{\Pi}}\right|_{\left(\omega_{r}, k_{r}, 1\right)}, \\
\dot{R}_{\omega} & =\left.\frac{\partial R\left(\omega, k_{n}, \varepsilon^{\Pi}\right)}{\partial \omega}\right|_{\left(\omega_{r}, k_{r}, 1\right)}, \\
R_{0} & =R\left(\omega_{r}, k_{r}, 1\right) .
\end{aligned}
$$

The growth will occur if $\delta$, the solution of Eq. (29) is imaginary and positive.

\section{Results and discussion}

By assuming no beam in the system, the dispersion relation is calculated by solving Eq. (27) numerically. The grating parameters are as follows: $R_{1}=240 \mu \mathrm{m}, R_{2}=400 \mu \mathrm{m}, l=80 \mu \mathrm{m}, d=160$ $\mu \mathrm{m}, a_{1}=400 \mu \mathrm{m}, a_{2}=480 \mu \mathrm{m}$ and the beam energy is $20 \mathrm{kev}$, corresponding to the parameters chosen by Y. Zhou et al. [16]. The effect of $\varepsilon_{r}$ on the dispersion relation has been shown in Fig. 2(a). It is clear that increasing the $\varepsilon_{r}$ results in smaller height of the dispersion relation. This means that the modes are propagating with smaller velocities in the system. The intersection points of beamwave also move down. In this figure, the corresponding growth rate for each curve is indicated by dash lines (of the same color). Maximum growth 
rates occur in the vicinity of the synchronous points $\left(\omega_{r}=k_{r} v_{0}\right)$, and have the values: $1.279,1.129$ and 1.065 for $\varepsilon_{r}=1,1.5$ and 2.7 , respectively. The influence of dielectric on the growth rate is clearer in Fig. 2(b), which indicates that dielectrics with higher relative permittivities cause smaller values for the growth rate. The grating parameters are considered in Fig. 3, Fig. 4 and Fig. 5, when $\varepsilon_{r}=2.7$.

In Fig. 3 the slot depth has been changed. As depth increases, the dispersion relation becomes flatter, indicating that the effect of grating is increasing. The normalized maximum growth rate happens when resonance between the beam and the modes is possible (the circles). So, in these points $\delta=1.192,1.066$ and 0.493 when $h=100$ $\mu \mathrm{m}, 160 \mu \mathrm{m}$ and $250 \mu \mathrm{m}$, respectively. Figure 4 indicates how dielectric thickness has an effect on growth rate. Again, lower frequency modes result from increasing dielectric thickness. However, this time the growth rate will increase by this effect: $\delta=0.959,1.232$ and 1.295 for $l=30 \mu \mathrm{m}, 80 \mu \mathrm{m}$ and $110 \mu \mathrm{m}$, respectively.

The effect of beam thickness $(\Delta)$ on growth rate is depicted in Fig. 5. First, increasing $\Delta$ causes the growth rate to rise. Its maximum value is 1.066 at $\Delta=80 \mu \mathrm{m}$. This happens because more electrons can participate in the beam wave interaction. Then the growth rate falls. This can be justified by the

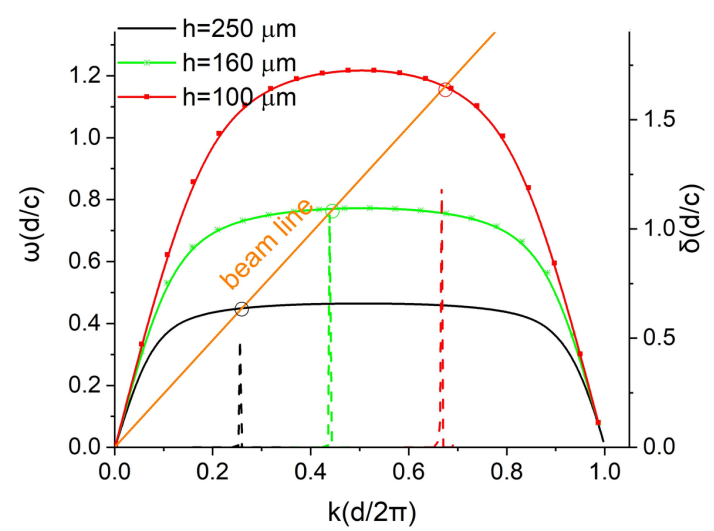

Figure 3: Comparison of dispersion relation for different grating heights, when $\varepsilon_{r}=2.7$. The growth rate corresponding to each frequency curve is plotted by dash lines, and maximum values are indicated at the circle points.

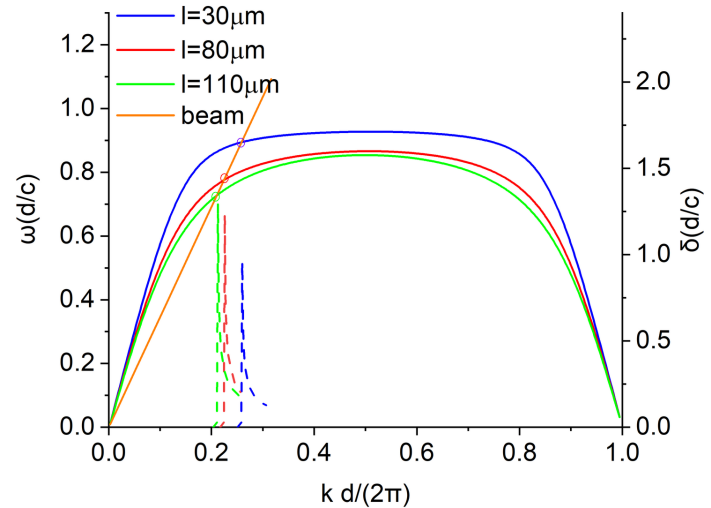

Figure 4: Comparison of the dispersion relations (solid lines) and the growth rates (dash lines) for different thicknesses of the dielectric $\varepsilon_{r}=2.7$.

fact that although the thickness is increasing, the electrons which are far from the grating contribute less to the interaction.

\section{Conclusions}

In this paper, a metallic cylindrical grating filled with a dielectric is proposed. The dispersion relation of the modes propagating in this configuration with an annular electron beam is derived. It is shown that the dielectric causes modes with smaller frequencies, in comparison with results when it is absent. Then, the growth rate of modes which

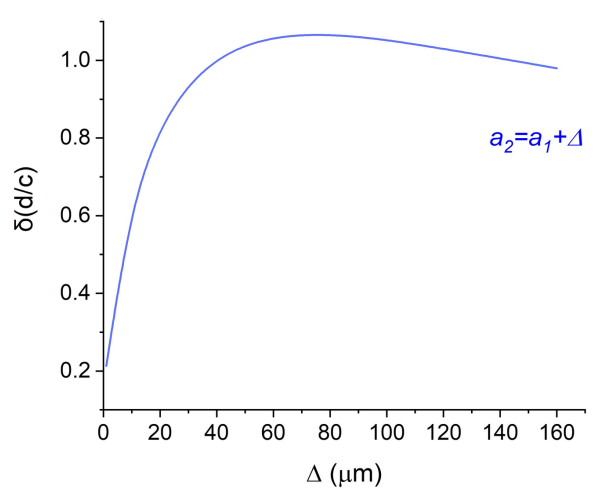

Figure 5: Dependence of the growth rate on beam thickness. $\varepsilon_{r}=2.7, R_{1}=240 \mu \mathrm{m}, R_{2}=400 \mu \mathrm{m}, l=80 \mu \mathrm{m}$, $d=160 \mu \mathrm{m}, a_{1}=400 \mu \mathrm{m}$. 
Papers in Physics, vol. 11, ART. 110007 (2019) / Z. Rezaei et al.

are in resonance with the beam is considered. It is found that the growth rate is under the influence of dielectric relative permittivity $\varepsilon_{r}$, the depth of the slots of the grating and the thickness of the dielectric (the width of the slots). A lower growth rate is the result of increasing the parameters of the dielectric relative permittivity and slot depth, and decreasing the thickness of the dielectric. Also, beam thickness can increase and decrease the growth rate, depending on its amount. As we can see, by changing the grating parameters, as well as dielectric permittivity and thickness, the growth rate and operating frequencies of the device can be controlled. So, it is possible to make SP-FELs with the desired frequencies and powers. These results can be of considerable interest for $\mathrm{THz}$ wave source research.

[1] B Maraghechi, B Farokhi, J E Willett, Theory of high-frequency waves in a coaxial plasma wave guide, Phys. Plasmas 6, 3778 (1999).

[2] S J Smith, E M Purcell, Visible light from localized surface charges moving across a grating, Phys. Rev. 92, 1069 (1953).

[3] L Schachter, A Ron, Smith-Purcell freeelectron laser, Phys. Rev. A 40, 876 (1989).

[4] J Urata, M Goldstein, M F Kimmitt, A Naumov, C Platt, J E Walsh, Superradiant SmithPurcell emission, Phys. Rev. Lett. 80, 516 (1998).

[5] Y Neo, H Shimawaki, $\mathrm{T}$ Matsumoto, $\mathrm{H}$ Mimura, Smith-Purcell radiation from ultraviolet to infrared using a Si field emitter, J. Vac. Sci. Technol. B 24, 924 (2006).

[6] K J Kim, S B Song, Self-amplified spontaneous emission in Smith-Purcell free-electron lasers, Nucl. Instrum. Methods Phys. Res. Sect. A, 475, 158 (2001).

[7] H L Andrews, C A Brau, Gain of a SmithPurcell free-electron laser, Phys. Rev. Spec. Top. Accel. Beams 7, 70701 (2004).
[8] H P Freund, T M Abu-Elfadl, Linearized field theory of a Smith-Purcell traveling wave tube, IEEE Trans. Plasma Sci. 32, 1015 (2004).

[9] D Li, Z Yang, K Imasaki, G-S Park, Particlein-cell simulation of coherent and superradiant Smith-Purcell radiation, Phys. Rev. Spec. Top. Accel. Beams 9, 040701 (2006).

[10] D N Klochkov, A I Artemyev, K B Oganesyan, Y V Rostovtsev, M O Scully, C-K Hu, The dispersion equation of the induced Smith-Purcell instability, Phys. Scr. T140, 014049 (2010).

[11] M Cao, W Liu, Y Wang, K Li, Threedimensional theory of Smith-Purcell freeelectron laser with dielectric loaded grating, J. Appl. Phys. 116, 103104 (2014).

[12] M Cao, W Liu, Y Wang, K Li, Dispersion characteristics of three dimensional dielectricloaded grating for terahertz Smith-Purcell radiation, Phys. Plasmas 21, 23116 (2014).

[13] W Liu, M Cao, Y Wang, K Li, Start current of dielectric-loaded grating in Smith-Purcell radiation, Phys. Plasmas 23, 33104 (2016).

[14] H P Bluem, R Jackson, J D Jarvis, A M M Todd, J Gardelle, P Modin, J T Donohue, First lasing from a high-power cylindrical grating Smith-Purcell device, IEEE Trans. Plasma Sci. 43, 9 (2015).

[15] S Hasegawa, K Ogura, T lwasaki, K Yambe, Smith-Purcell radiation based on cylindrical surface waves, Fusion Sci. Technol. 63, 259 (2017).

[16] Y Zhou, Y Zhang, S Liu, Electron-beam-driven enhanced terahertz coherent Smith-Purcell radiation within a cylindrical quasi-optical cavity, IEEE Trans. THz Sci. Technol. 6, 262 (2016). 